Europhysics Letters

PREPRINT

\title{
Novel low-energy collective excitation at metal surfaces
}

\author{
V. M. Silkin ${ }^{1}$, A. García-Lekue ${ }^{2}$, J. M. Pitarke ${ }^{1,2}$, E. V. Chulkovov $^{1,3}$, E. \\ ZAREMBA $^{4}$, and P. M. ECHENIQUE ${ }^{1,3}$ \\ 1 Donostia International Physics Center (DIPC) and Centro Mixto CSIC-UPV/EHU - \\ Manuel de Lardizabal Pasealekua, E-20018 Donostia, Basque Country, Spain \\ 2 Materia Kondentsatuaren Fisika Saila, Zientzi Fakultatea, Euskal Herriko Unibertsi- \\ tatea - 644 Posta kutxatila, E-48080 Bilbo, Basque Country, Spain \\ 3 Materialen Fisika Saila, Kimika Fakultatea, Euskal Herriko Unibertsitatea - 1072 \\ Posta kutxatila, E-20018 Donostia, Basque Country, Spain \\ 4 Department of Physics, Queen's University - Kingston, Ontario, Canada KYL $3 N 6$
}

\begin{abstract}
PACS. 71.45.Gm - .
PACS. 73.20.At -

PACS. 73.50.Gr - .
\end{abstract}

\begin{abstract}
A novel collective excitation is predicted to exist at metal surfaces where a two-dimensional surface-state band coexists with the underlying three-dimensional continuum. This is a low-energy acoustic plasmon with linear dispersion at small wave vectors. Since new modern spectroscopies are especially sensitive to surface dynamics near the Fermi level, the existence of surface-state induced acoustic plasmons is expected to play a key role in a large variety of new phenomena and to create situations with potentially new physics.
\end{abstract}

The long-range nature of the Coulomb interaction between electrons in a three-dimensional (3D) metal is known to yield collective behaviour, manifesting itself in the form of plasma oscillations [1]. These collective charge-density oscillations are a fundamental property of metals, and are known to be of basic importance in the interpretation of a large variety of experiments.

In 1957 Ritchie introduced the idea of surface plasmons [2], which are collective oscillations bound to a metal surface. In the long-wavelength limit, the energy of the surface plasmon is given by $\hbar \omega_{s}=\hbar \omega_{p} / \sqrt{2}$, where $\omega_{p}=\left(4 \pi n e^{2} / m_{e}\right)^{1 / 2}$ is the bulk-plasma frequency, $n$ being the electron density and $m_{e}$ the electron mass. The concept of surface plasmons has played a significant role in a variety of areas of fundamental and applied research, from surface dynamics [3,4] to surface-plasmon microscopy [5], surface-plasmon resonance technology [6], and a wide range of photonic applications [7-9].

Another collective excitation arises in a two-dimensional (2D) gas of electrons confined to a plane. In this case, the $2 \mathrm{D}$ plasmon has energy $\hbar \omega_{2 D}=\hbar\left(2 \pi n^{2 D} e^{2} q / m_{e}\right)^{1 / 2}\left(n^{2 D}\right.$ is the $2 \mathrm{D}$ electron density), which goes to zero as $q^{1 / 2}$ as the $2 \mathrm{D}$ wave number $q$ tends to zero [10]. The 2D plasmon was first observed in artificially structured semiconductors [11] and more recently in a metallic surface-state band on a silicon surface [12].

(C) EDP Sciences 
Since the typical energy $\hbar \omega_{p}$ of a three-dimensional (3D) plasmon is a few electronvolts, thermal excitation of bulk and surface plasmons is improbable and the electronic properties near the Fermi level cannot be influenced by these excitations. Plasmons in a 2D electron gas have lower energies, but they still do not affect electron-hole (e-h) and phonon dynamics near the Fermi level due to their square-root dependence on the wave vector. Much more effective than ordinary $3 \mathrm{D}$ or $2 \mathrm{D}$ plasmons in mediating, e.g., superconductivity would be the so-called acoustic plasmons with sound-like long-wavelength dispersion, which have spurred over the years a remarkable interest and research activity [13].

Pines originally suggested that acoustic plasmons could be realized in the collective motion of a system of two types of electronic carriers [14]. The possibility of having a longitudinal acoustic mode in a metal-insulator-semiconductor structure was anticipated by Chaplik [15]. Chaplik considered a simplified model in which a 2D electron gas is separated from a semiinfinite metal. He found that the screening of valence electrons in the metal changes the 2D plasmon energy from the square-root behaviour to a linear dispersion, which was also discussed by Gumhalter [16] in his study of transient interactions of surface-state e-h pairs at metal surfaces. However, acoustic plasmons were only expected to exist for spatially separated plasmas, as pointed out by Das Sarma and Madhukar [17]. Acoustic plasma oscillations were observed in two-dimensionally confined and spatially separated multicomponent structures such as quantum wells and heterojunctions [18], and were then proposed as possible candidates to mediate the attractive interaction leading to the formation of Cooper pairs in high- $T_{c}$ superconductors $[19,20]$.

In this Letter, we show that in metals with a partially occupied surface-state band the dynamical screening at the surface provides a mechanism for the existence of a new acoustic collective mode whose energy exhibits a linear dependence on the $2 \mathrm{D}$ wave number $q$. We consider the example of the $\mathrm{Be}(0001)$ surface, with no $d$ bands, which presents a very-highly populated two-dimensional Shockley surface-state band of the $s$ - $p_{z}$ symmetry [21-23], and as such is an ideal candidate to investigate the nature of the dynamical screening in a $2 \mathrm{D}$ electron gas that is immersed in a 3D system. The novel result of our detailed band-structure calculations, in which transitions between the surface-state band and all other states of the semi-infinite metal are fully included, is that in a real metal surface where a partially occupied quasi-2D surface-state band coexists in the same region of space with the underlying 3D continuum, a well-defined surface acoustic plasmon exists.

First of all, we calculate the eigenfunctions and eigenvalues of a one-dimensional singleparticle hamiltonian that describes the main features of the surface band structure [24, 25]. Then we evaluate the dynamical density-response function $\chi^{0}\left(z, z^{\prime} ; q, \omega\right)$ of non-interacting electrons, and solve an integral equation to obtain the interacting density-response function $\chi\left(z, z^{\prime} ; q, \omega\right)$ in the random-phase approximation (RPA) which is known to be exact in the $q \rightarrow 0$ limit [26]. $\quad \chi\left(z, z^{\prime} ; q, \omega\right)$ contains both bulk and surface states and accounts for all possible transitions between them.

Assuming translational invariance in the plane of the surface, the Fourier components of the induced density satisfy the equation

$$
\delta n(z ; q, \omega)=\int d z^{\prime} \chi\left(z, z^{\prime} ; q, \omega\right) \phi^{e x t}\left(z^{\prime} ; q, \omega\right)
$$

and the collective oscillations created by an external potential of the form

$$
\phi^{e x t}(z ; q, \omega)=-(2 \pi / q) \mathrm{e}^{q z}
$$



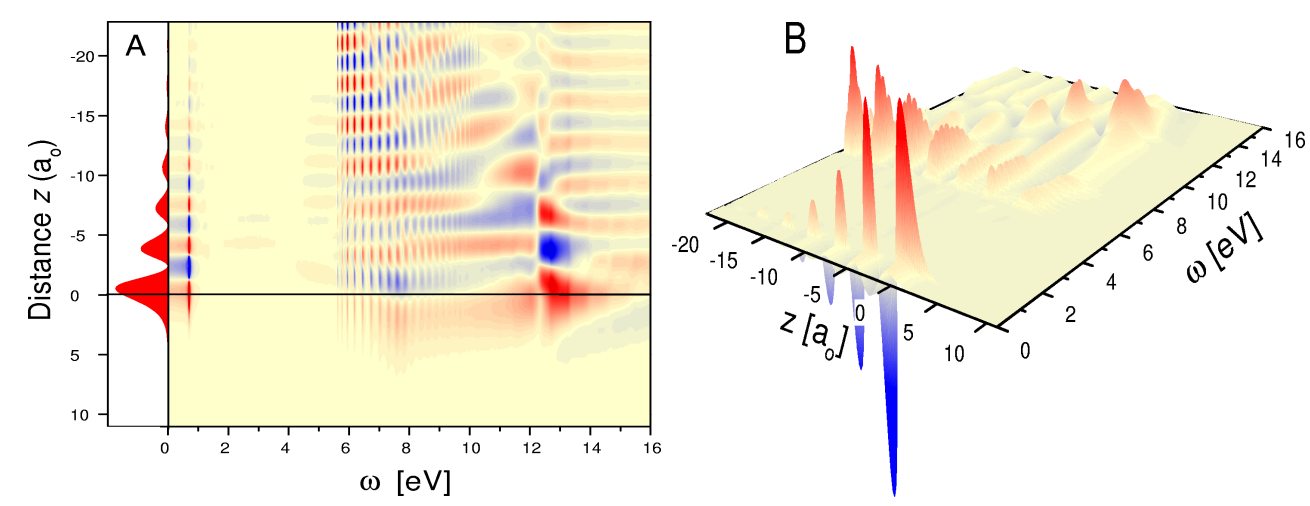

Fig. 1 - RPA calculation of the imaginary part of the electron density induced in $\operatorname{Be}(0001)$, as obtained from eqs. (1) and (2) for the wave number $q=0.05$ versus the energy $\omega$ and the coordinate $z$ normal to the surface. The crystal edge $(z=0)$ is chosen to be located half a lattice spacing beyond the last atomic layer, and $z<0$ corresponds to the interior of the solid. (a) 2D plot of $\operatorname{Im}[\delta n(z ; q, \omega)] / \omega$. Positive and negative values are represented by red and blue colours, respectively, more intense colours corresponding to larger absolute values. The probability density of the Shockley surface state is represented on the left-hand side (red shaded areas), as a function of the $z$ coordinate. (b) $3 \mathrm{D}$ plot of $\operatorname{Im}[\delta n(z ; q, \omega)] / \omega$.

can be traced to the peaks of the imaginary part of the surface response function $[27,28]$

$$
\operatorname{Im}[g(q, \omega)]=\int d z \mathrm{e}^{q z} \operatorname{Im}[\delta n(z ; q, \omega)]
$$

In fig. [1 we show our full RPA calculation of the imaginary part of the electron density $\delta n(z ; q, \omega)$ induced in a $\operatorname{Be}(0001)$ surface by the external electrostatic potential of eq. (2) with $q=0.05$. This figure exhibits two distinct spectral features. The first feature occurs at the surface-plasmon frequency of valence $\left(2 s^{2}\right)$ electrons in $\mathrm{Be}\left(\hbar \omega_{s} \sim 13 \mathrm{eV}\right)$, where $\operatorname{Im}[\delta n]$ exhibits a strong maximum at the surface $(z=0)$ and pronounced Friedel oscillations in the interior of the solid $(z<0)$; these are the main characteristics of the ordinary monopolar surface-plasmon mode, which would also be present in a semi-infinite free-electron gas (FEG) of bulk density equal to that of valence electrons in Be. In addition, however, very localized local maxima are visible near the surface at an energy of $0.6 \mathrm{eV}$, which correspond to a lowenergy collective excitation that would be absent in a semi-infinite FEG. A comparison with the probability density of the partially occupied Shockley surface state, which is shown by red shaded areas on the left-hand side of fig. 1h, clearly indicates that the low-energy collective excitation originates from this $2 \mathrm{D}$ surface-state band. Nevertheless, such a $2 \mathrm{D}$ electron gas alone would only support a plasmon that for $q=0.05$ has energy $\omega_{2 D}=2.7 \mathrm{eV}$, well above the low-energy excitation that is visible in fig. 目, and it is only the combination of the strongly localized 2D surface-state band with 3D bulk states which allows the formation of this new mode.

Figure 2 shows the imaginary part of the surface-response function $g(q, \omega)$ of $\operatorname{Be}(0001)$, as obtained from eq. (3) for increasing values of $q$. As follows from the figure, the excitation spectra is dominated by the conventional surface plasmon at $\hbar \omega_{s} \sim 13 \mathrm{eV}$, which can be traced to the characteristic pole that the surface response function $g(q, \omega)$ of a bounded 3D electron gas exhibits at this energy, and a well-defined low-energy peak with linear dispersion. Figure 2 clearly shows that the low-frequency mode has linear dispersion, with the sound velocity 


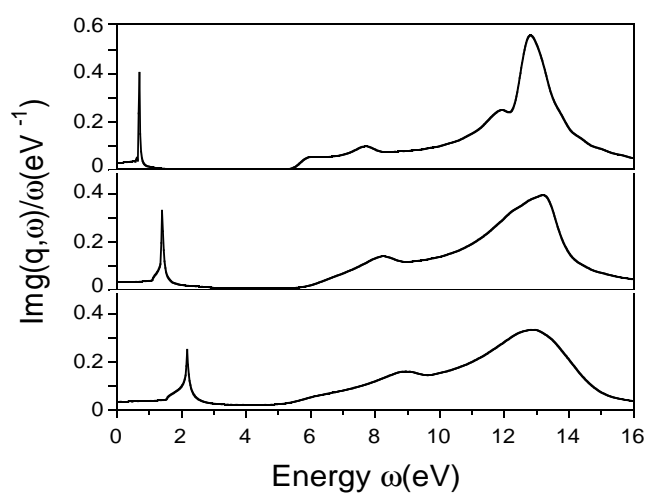

Fig. 2 - Energy-loss function $\operatorname{Im}[g(q, \omega)] / \omega$ of $\operatorname{Be}(0001)$ versus the excitation energy $\omega$, as obtained from eq. (3) for various values of the wave number: $q=0.05$ (top panel), 0.1 (middle panel), and 0.15 (bottom panel), in units of the inverse Bohr radius $a_{0}^{-1}$. The peaks are dictated by the corresponding poles of the surface response function $g(q, \omega)$. In the long-wavelength limit $(q \rightarrow 0), g(q, \omega)$ is simply the total electron density induced by the potential of eq. (2).

being very close to the $2 \mathrm{D}$ Fermi velocity $v_{F}^{2 D}$.

In fig. [3 we show the energy of the low-energy mode in Be(0001) versus $q$ (red solid line), as derived from the maxima of our calculated surface loss function $\operatorname{Im}[g(q, \omega)]$, together with the well-defined plasmon energies that we obtain when only the surface-state band is considered in the evaluation of the dynamical density-response function (blue solid line). While the plasmon energies of electrons in the isolated surface-state band nicely reproduce in the long-wavelength limit the conventional plasmon dispersion $\omega_{2 D}$ of electrons in a 2D electron gas (green dashed line), the combination of this surface-state band with the underlying 3D system yields a new distinct mode whose energy lies just above the upper edge $\omega_{u}^{2 D}=v_{F}^{2 D} q+q^{2} / 2$ of the $2 \mathrm{D}$ e-h pair continuum (shaded area). At small $q$, this surface acoustic mode has linear dispersion,

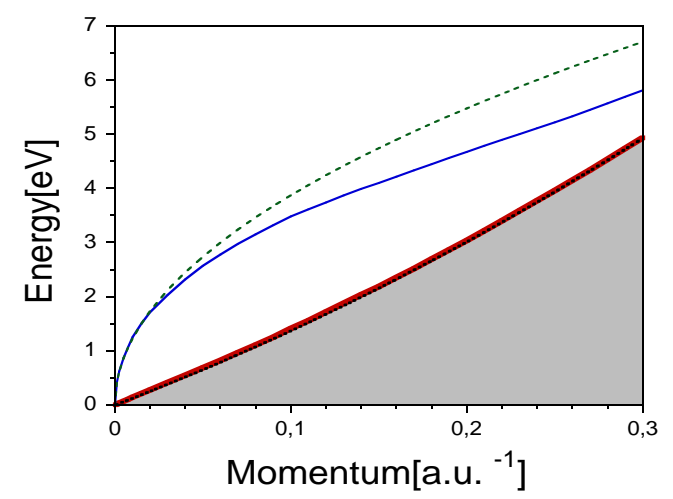

Fig. 3 - Dispersion of the new low-energy collective mode that is visible in fig. 2 (red solid line), together with the result we obtain when bulk states are omitted in the evaluation of the energy-loss function (blue solid line) and the conventional plasmon dispersion $\omega_{p}^{2 D}=v_{F}^{2 D} \sqrt{q}$ of electrons in a purely 2D electron gas with $v_{F}^{2 D}=0.45 v_{0}$ (green dashed line). The dotted line represents the upper edge $\omega_{u}^{2 D}=v_{F}^{2 D} q+q^{2} / 2$ of the $2 \mathrm{D}$ e-h pair continuum (shaded area). We note that momentum and energy conservation prevents $2 \mathrm{D}$ e-h pairs to be produced for energies above $\omega_{u}^{2 D}$. 
with the sound velocity being very close to the Fermi velocity of the 2D surface-state band.

Figures 13 show that acoustic surface plasmons are low-energy collective oscillations appropriate to very localized slow Shockley electrons with Fermi velocity $v_{F}^{2 D}<v_{0}$ plus their associated cloud of swift bulk electrons with $v_{F}^{3 D} \sim v_{0}$. These excitations cannot occur at the characteristic energy of the usual 2D plasmons, where Landau damping would be possible by virtue of $3 \mathrm{D}$ e-h pair creation. Instead, an acoustic plasmon mode occurs at energies that follow closely the upper edge of the 2D e-h pair continuum.

In conclusion, we have shown that the combination of a localized surface-state $2 \mathrm{D}$ band of slow electrons with a bulk 3D electron gas of fast electrons $\left(v_{F}^{3 D}>v_{F}^{2 D}\right)$, which takes place in the (0001) surface of Be, leads to the formation of a novel low-energy collective excitation. This new acoustic mode has linear dispersion at small wave vectors, with the sound velocity being very close to the Fermi velocity of the $2 \mathrm{D}$ surface-state band. A similar mode is also found to exist at the (111) surfaces of the noble metals [29]. We hope that our prediction of acoustic surface plasmons will stimulate new experiments in this direction.

Partial support by the University of the Basque Country, the Basque Unibertsitate eta Ikerketa Saila, the Spanish MCyT, and the Max Planck Research Award Funds is gratefully acknowledged.

\section{REFERENCES}

[1] PINES D. and BOHM D., Phys. Rev., 85 (1952) 338.

[2] RITCHIE R. H., Phys. Rev., 106 (1957) 874.

[3] ISHIDA H., SHIMA N. and TSUKADA M., Phys. Rev. B, 32 (1985) 6246.

[4] ROCCA M., Surf. Sci. Rep., 22 (1995) 1.

[5] ROTHENHAUSLER B. and KNOLL W., Nature, 332 (1988) 615.

[6] SChUSTER S. C., SWANSON R. V., AlEX L. A., BOURRET R. B. and SIMON M. I., Nature, 365 (1993) 343.

[7] EBBESEN T. W., LEZEC H. J., GHAEMI H. F., ThiO T. and WOLFF P. A., Nature, 391 (1998) 667.

[8] PENDRY J. B., Science, 285 (1999) 1687.

[9] LEZEC H. J., DEGIRON A., DEVAUX E., LINKE R. A., MARTIN-MORENO L., GARCIAVIDAL F. J. and EBBESEN T. W., Science, 297 (2002) 820.

[10] STERN F., Phys. Rev. Lett., 18 (1967) 546.

[11] ALlen, Jr. S. J., TSUI D. C. and LOGAN R. A., Phys. Rev. Lett., 38 (1977) 980.

[12] NAGAO T., HELDEBRANDT T., HENZLER M. and HASEGAVA S., Phys. Rev. Lett., 86 (2001) 5747.

[13] MARCH N. H. and TOSI M. P., Adv. Phys., 44 (1995) 299.

[14] PINES D., Can. J. Phys., 34 (1956) 1379.

[15] CHAPLIK A. V., Zh. Eksp. Teor. Fiz., 62 (1972) 746 [Sov. Phys.-JETP, 35 (1972) 395].

[16] GUMHALTER B., Surf. Sci., 518 (2002) 81.

[17] DAS SARMA S. and MADHUKAR A., Phys. Rev. B, 23 (1981) 805.

[18] Olego D., PINCZUK A., GOSSARD A. C. and WIEGMANN W., Phys. Rev. B, 25 (1982) 7867.

[19] RUVAlDS J., Phys. Rev. B, 35 (1987) 8869.

[20] BILl A., MORAWITZ H. and KREZIN V. Z., Phys. Rev. B, 66 (2002) 100501.

[21] BARTYNSKY R. A., JENSEN E., GUSTAFSSON T. and PlumMER E. W., Phys. Rev. B, 32 (1985) 1921

[22] CHULKOV E. V., SILKIN V. M. and SHIRYKALOV E. N., Surf. Sci., 188 (1987) 287.

[23] On Be(0001), a Shockley surface state with a binding energy of $2.8 \mathrm{eV}$ at $\bar{\Gamma}$ exists in the $\Gamma_{3}^{+}-\Gamma_{4}^{-}$ bulk band gap. This surface state disperses with momentum parallel to the surface, thereby forming a surface-state band with a $2 \mathrm{D}$ Fermi energy $E_{F}^{2 D}=2.8 \mathrm{eV}$. If one takes the effective 
EUROPHYSICS LETTERS

mass of surface-state electrons to be equal to the free-electron mass $m_{e}$, the $2 \mathrm{D}$ Fermi velocity is found to be $v_{F}^{2 D}=0.45 v_{0}$, with $v_{0}=e^{2} / \hbar$ being the Bohr velocity.

[24] CHULKOV E. V., SILKIN V. M. and ECHENIQUE P. M., Surf. Sci., 437 (1999) 330.

[25] EChEnique P. M., PITARKE J. M., CHUlKOV E. V. and RUBiO A., Chem. Phys., 251 (2000) 1.

[26] PINES D., Elementary excitations in solids (W. A. Benjamin, Inc., New York) 1963.

[27] PERSSON B. N. J. and ZAREMBA E., Phys. Rev. B, 31 (1985) 1863.

[28] LIEBSCH A., Electronic Excitations at Metal Surfaces (Plenum, New York) 1997.

[29] SILKIN V. M., GARCIA-LEKUE A., PITARKE J. M., CHULKOV E. V., ZAREMBA E., and ECHENIQUE P. M. (unpublished). 